\title{
P8 O-GlcNAcylation Increases Constriction in Common Carotid Artery of Senescent-Accelerated Female Mice
}

\author{
Tiago J. Costa ${ }^{1, *}$, Josiane F. Silva ${ }^{1}$, Renée de Nazaré O. da Silva ${ }^{2}$, Paula R. Barros ${ }^{2}$, Juliana Bolsoni ${ }^{1}$, \\ Daniel Rodrigues ${ }^{1}$, Eliana H. Akamine ${ }^{2}$, Rita Tostes ${ }^{1}$ \\ ${ }^{1}$ Department of Pharmacology, Ribeirao Preto Medical School, University of Sao Paulo, Brazil \\ ${ }^{2}$ Department of Pharmacology, Institute of Biomedical Sciences, University of Sao Paulo, Brazil
}

\begin{abstract}
The glycosylation with $\mathrm{O}$-linked- $\mathrm{N}$-acetylglucosamine $(\mathrm{O}-\mathrm{GlcNAc})$ is an enzymatic process found on nuclear and cytoplasmic proteins. Augmented levels of $O$-GlcNAc-modified proteins have been associated to cardiovascular disease (CD) in male. However, the association of $O$-GlcNAc and $\mathrm{CD}$ in females it's not understood. Increased risk of $\mathrm{CD}$ in women is linked to menopause and occurs about 10 years later than in men. We tested the hypothesis that aging in females promotes vascular dysfunction, which is aggravated by augmented O-GlcNAc. We used 8-month-old female senescence-mice (SAMP8) and control (SAMR1). After euthanasia, the carotid artery (CA) was carefully removed and cleaned. The segments of CA were incubated for 24h in DMEM containing vehicle/or/Thiamet $\mathrm{G}(10-6 \mathrm{M})$, a potent inhibitor of the $O$-GlcNAcase. Vessels from both groups were carefully mounted as ring preparations in standard organ chambers. Concentration-response curves to U46619 and phenylephrine (PE) (10-10 to $10-4 \mathrm{M})$ were performed in vessels with endothelium in the presence or not of a COX inhibitor. Results are presented as mean \pm SEM, $n=6$. Statistical analysis: ANOVA One-way, followed Bonferroni. $p<0.05$. CA of SAMP8 (Rmax127 \pm 10 ) exhibited increased PE-induced vasoconstriction, compared to SAMR1 (Rmax77 \pm 5 ), which was abolished in the presence of the COX inhibitor. mRNA expression of COX-1/COX-2 was similar in both groups, but the eNOS decreased in CA of SAMP8. O-GlcNAcylation increased vasoconstriction in SAMP8 but not in SAMR1 female mice, indicating an important correlation between $O$-GlcNAcylation and aging process. Conclusion: the senescence is associated with vascular dysfunction mediated by abnormal activity of COX and eNOs, which is aggravated by augmented O-GlcNAcylation. FAPESP (2017/25116-2).
\end{abstract}

(c) 2019 Association for Research into Arterial Structure and Physiology. Publishing services by Atlantis Press International B.V. This is an open access article distributed under the CC BY-NC 4.0 license (http://creativecommons.org/licenses/by-nc/4.0/) 